REGARDS

SUR L'ECONOMIE ALLEMAND

BULLETIN ECONOMIQUE DU CIRAC
Regards sur l'économie allemande

Bulletin économique du CIRAC

$75 \mid 2006$

Varia

\title{
Une confiance retrouvée
}

Isabelle Bourgeois

\section{OpenEdition}

Journals

Édition électronique

URL : https://journals.openedition.org/rea/824

DOI : $10.4000 /$ rea. 824

ISBN : 978-2-8218-0846-1

ISSN : 1965-0787

Éditeur

CIRAC

Édition imprimée

Date de publication : 1 mars 2006

Pagination : 3-4

ISSN : 1156-8992

Référence électronique

Isabelle Bourgeois, "Une confiance retrouvée », Regards sur l'économie allemande [En ligne], 75 | mars 2006, mis en ligne le 06 juin 2008, consulté le 28 juin 2022. URL : http://journals.openedition.org/rea/ 824 ; DOI : https://doi.org/10.4000/rea.824 


\section{Une confiance retrouvée}

Depuis le début de l'année, les signes d'embellie se multiplient au point que les experts attendent désormais un taux de croissance de $2 \%$ pour 2006. Certes, le ministre fédéral de l'Economie n'avance qu'un prudent 1,4\% ; mais son estimation remonte à la fin janvier. Or depuis l'été dernier, l'indice ifo du climat des affaires ne cesse de grimper; et ces dernières semaines, la confiance se diffuse nettement dans toutes les activités, y compris dans le commerce de détail et même le BTP ; elle s'étend à l'est comme à l'ouest. Quant aux PME, dont le moral est souvent à la traîne des grandes entreprises, leur optimisme renoue actuellement avec son niveau record de 2000.

La solide confiance des acteurs économiques à la fois dans la situation présente et dans l'avenir se fonde sur la bonne tenue d'un certain nombre d'indicateurs. Les exportations ont une nouvelle fois battu leur propre record en 2005 : elles avaient progressé de $7,5 \%$ pour dépasser 786 milliards $€$, et le solde commercial s'était élevé à 160,5 milliards € (Destatis). L'UE reste le premier débouché pour l'Allemagne (près de 497 milliards $€$ ) bien que les exportations y aient connu une hausse $(+6,9 \%)$ inférieure à celles des ventes vers les pays tiers $(+8,5 \% ; 287,6$ milliards $€)$. Au total, c'est encore une fois le solde commercial qui a tiré la croissance allemande : il a contribué à hauteur de 0,7 points à une croissance du PIB de $+0,9 \%$ avant correction de l'effet calendrier $(+1,1 \%$ après). La Fédération de l'industrie BDI, se fondant sur le triple effet stimulant d'un taux de change $€ / \$$ favorable, d'une forte demande mondiale et d'une reprise de l'investissement en Europe au second semestre, s'attend à une hausse des exportations encore plus soutenue en 2006.

Les entrées en commande dans l'industrie enregistrées en janvier confirment cet optimisme. Leur hausse de $+1,4 \%$ s'explique partiellement par quelques opérations d'envergure, mais vient après un mois de décembre où le recul a été plus léger $(-0,3 \%)$ que prévu (contre $-1,6 \%$ avant correction). Plus significatif : sur la période décembre/janvier, les commandes ont augmenté de $+1,1 \%$ par rapport à la même période de l'année précédente. Cette bonne tenue est largement imputable aux biens d'investissement $(+4,3 \%)$, tirés par une demande étrangère en hausse de $+7,3 \%$. Le secteur le plus florissant est la construction mécanique, troisième branche d'Allemagne, exportatrice par excellence. Elle est dopée depuis trois ans par la demande des Etats-Unis comme de l'Europe, mais aussi par les marchés émergents et le boom des commandes des pays de I'OPEP. Plus important : elle n'a pas encore épuisé le potentiel qui réside dans la vente de services liés aux installations ou machines. Cette branche où dominent les PME, dont le taux d'exportation a frisé les $74 \%$ l'an dernier (la valeur des exportations, de 107 milliards $€$, avait crû de $+8 \%$ ) et dont la valeur de la production a atteint 145 milliards $€(+4,4 \%)$, est l'un des principaux moteurs de l'économie allemande. Si 2006 s'avère aussi favorable qu'elle s'annonce, le secteur enregistrerait une croissance cumulée de $+12 \%$ sur la période 20042006. Car malgré une demande mondiale susceptible de se ralentir dans le courant de l'année 2006, la Fédération VDMA n'en constate pas moins depuis le début de l'année une hausse des commandes domestiques.

II semblerait en effet que nombre d'entreprises allemandes se décident à investir ; elles en expriment clairement l'intention dans les sondages. II est vrai que l'amélioration du régime des amortissements les y incite. Mais pour l'instant, elles hésitent encore à étendre leurs capacités, préférant les renouveler après une longue phase où elles avaient rationalisé leur production, consolidé leur base financière, puis accumulé des stocks. Plus généralement, les investissements bruts en biens d'équipement ont été en hausse ( $+0,7 \%$; Destatis), pour la troisième fois consécutive, au cours du $3^{\text {ème }}$ trimestre 2005. Ils sont tirés par la construction $(+1,2 \%)$, ce qui sort provisoirement le BTP du marasme où il est
Un solde commercial record

Des commandes de biens d'investissement en hausse

Hausse des investissements en biens d'équipement 
Hausse de la demande intérieure

Une politique de consolidation budgétaire jugée contradictoire plongé depuis une dizaine d'années. Si les entreprises investissent, les ménages pour leur part, ont eux aussi démultiplié leurs commandes immobilières afin de bénéficier de la prime d'accession à la propriété avant sa suppression au $1^{\mathrm{er}}$ janvier 2006. Dans l'ensemble, constate l'institut IfW de Kiel, les investissements d'équipement sont orientés à la hausse, et ce depuis 2004. C'est sur cette tendance que l'IfW et le RWI fondent la révision à la hausse de leurs prévisions de croissance 2006 , établie à respectivement $2,1 \%$ et $1,8 \%$.

La demande intérieure, qui donne des signes de frémissement depuis quelque temps, devrait se consolider : elle pourrait même enregistrer, pour la première fois depuis 2000, une hausse, que l'IfW établit à $+1,2 \%$ pour 2006. A moyen terme, étant donné la propension des entreprises à investir et à embaucher - à condition toutefois que la progression salariale reste modérée -, cette tendance devrait se poursuivre. Mais la progression de la demande intérieure ne dépassera guère $1 \%$, explique l'institut. Le cadre réservé aux activités ne s'est en effet pas encore significativement amélioré. Les perspectives quant à l'évolution des revenus des ménages sont, elles, brouillées. D'une part, par les tensions persistantes sur le marché de l'emploi : le nombre de chômeurs amorce sa décrue, mais reste élevé ; il devrait tomber de 5,3 millions en février à quelque 4,6 millions en moyenne sur l'ensemble de l'année. D'autre part, la fourchette des revenus s'élargit vers le bas : près de 7 millions d'Allemands ( $9 \%$ de la population active ; BA) sont bénéficiaires de l'aide $A L G ~ I I$; travail temporaire et petits boulots se diffusent largement. Ajoutée à la modération salariale, cette tendance s'est traduite par une chute du revenu réel par actif : en 2005, la légère hausse du revenu brut $(+0,5 \%)$ et net $(+0,9 \%)$ a été absorbée par l'inflation (+2\% ; Destatis), tirée pour près de la moitié par le coût de l'énergie.

Les perspectives des ménages sur l'évolution de leurs revenus sont brouillées d'autre part par une politique de consolidation budgétaire perçue comme contradictoire. Si Peer Steinbrück a annoncé la volonté de l'Allemagne de respecter les critères de déficit de Maastricht en 2007, c'est sa manière d'y parvenir qui fait débat; les experts auraient préféré une réduction des dépenses publiques (elles sont en hausse de $+0,7 \%$ ) à l'augmentation de la TVA programmée pour 2007 (elle passera de $16 \%$ à $19 \%$ ). L'IfW rappelle que le volume des subventions à l'économie atteint près de 145 milliards $€$ (soit 6,5\% du PIB). Or ces aides sont financées par l'impôt ; les ramener à de plus justes proportions reviendrait à réduire le poids des prélèvements et donc à lever l'un des principaux handicaps à la dynamique de croissance. Les critiques au gouvernement sont avivées depuis la publication par Destatis à la fin février du déficit de 2005 ; il n'est plus que de 3,3\% (au lieu de 3,5\%). Si la croissance se confirme, ce taux pourrait tomber à $3 \%$ dès cette année. Or la politique du gouvernement fédéral est justement de soutenir la croissance en 2006, avant d'aborder une année 2007 chargée de réformes lourdes ; il avait adopté à cet effet un programme de relance de 25 milliards $€$ (voir REA 74/05), partiellement financé par des privatisations, et surtout par l'endettement (38 milliards $€$ ). Seule une croissance solide permettra au gouvernement fédéral de ramener, comme prévu, le déficit à $2,5 \%$ du PIB en 2007. Mais les instituts s'attendent en 2007 à un tassement du fait que la TVA renchérira le facteur travail, que la BCE vient d'augmenter son taux directeur et que l'activité mondiale devrait se ralentir...

Au PRINTEMPS 2006, LA CONFIANCE des Allemands est entretenue par la forte demande mondiale, le regain de compétitivité des entreprises, la crédibilité de l'action du gouvernement de grande coalition, sans oublier l'approche du Mondial de football. L'optimisme est tangible : les entreprises investissent et les ménages se remettent à consommer. Mais cette confiance engage le gouvernement, puisqu'elle comporte un appel explicite à la poursuite des grands chantiers de réformes. S'il confirme ces attentes dès que les élections régionales de la fin mars seront passées, le courage avec lequel l'opinion envisage un avenir qui s'annonce difficile pourra perdurer.

Isabelle Bourgeois (10-03-2006) 